\title{
Valganciclovir and bevacizumab for recurrent glioblastoma: A single-institution experience
}

\author{
CHENGWEI PENG $^{1}$, JIALING WANG ${ }^{2}$, JARRED P. TANKSLEY ${ }^{1}$, BRET C. MOBLEY ${ }^{3}$, \\ GREGORY D. AYERS ${ }^{4}$, PAUL L. MOOTS ${ }^{1}$ and STEPHEN W. CLARK ${ }^{1}$ \\ Departments of ${ }^{1}$ Neurology, ${ }^{2}$ Neurosurgery and ${ }^{3}$ Pathology; ${ }^{4}$ Center for Quantitative Sciences, \\ Vanderbilt University School of Medicine, Nashville, TN 37232, USA
}

Received July 31, 2015; Accepted November 24, 2015

DOI: $10.3892 / \mathrm{mco} .2015 .692$

\begin{abstract}
Prolonged treatment with adjuvant valganciclovir has been shown in one retrospective study to exert a significant effect on overall survival (OS) in newly diagnosed patients with glioblastoma multiforme (GBM). However, studies evaluating the effectiveness of valganciclovir in the treatment of recurrent GBM have not been performed. We evaluated the effect of valganciclovir in the recurrent setting in combination with bevacizumab therapy. A retrospective analysis was performed on patients treated for recurrent GBM with off-label valganciclovir and bevacizumab at Vanderbilt University. We identified 13 patients who received valganciclovir plus bevacizumab at some point during their treatment, 8 of whom were started on valganciclovir and bevacizumab concurrently upon first recurrence, whereas 5 had valganciclovir added to their bevacizumab regimen prior to a second recurrence. Of these patients, 12 were pathologically confirmed to have GBM, and 1 patient was diagnosed with gliosarcoma. We also identified an institutional cohort of 50 patients who had not been exposed to valganciclovir, but were treated with bevacizumab for first recurrence. The progression-free survival (PFS) at 6 months (PF6) and median OS (mOS) in the valganciclovir plus bevacizumab group was $62 \%$ and 13.1 months, respectively, for all 13 patients, and 50\% and 11.3 months, respectively, for the 8 concurrently treated patients. In the institutional bevacizumab cohort, the PF6 and mOS were $34 \%$ and 8.7 months, respectively. In this retrospective analysis, valganciclovir in combination with bevacizumab exhibited a trend toward improved survival in patients with recurrent GBM. However, given the small sample size and the retrospective nature of this study, a larger prospective study is required to confirm these results.
\end{abstract}

Correspondence to: Dr Stephen W. Clark, Department of Neurology, Vanderbilt University School of Medicine, 1161 21st Avenue South, Nashville, TN 37232, USA

E-mail: stephen.w.clark@vanderbilt.edu

Key words: valganciclovir, bevacizumab, glioblastoma multiforme, cytomegalovirus, Valcyte, Avastin

\section{Introduction}

Glioblastoma multiforme (GBM) is the deadliest primary brain tumor. The standard-of-care for treating GBM includes surgical resection followed by chemoradiation with temozolomide and adjuvant temozolomide (1). Invariably, these tumors progress after or during standard therapy, and treatment at recurrence involves the use of the anti-angiogenic antibody, bevacizumab. Once GBM progresses, survival is dismal and there is an urgent need for effective therapies.

Recently, there has been significant interest and some controversy regarding the use of the antiviral agent valganciclovir for the treatment of newly diagnosed GBM patients. Initially, Stragliotto et al (2) reported a randomized, double-blind, placebo-controlled study in which newly diagnosed GBM patients received either valganciclovir or placebo in addition to standard therapy for 6 months; they reported no statistically significant difference in overall survival (OS) in the valganciclovir arm compared with placebo. However, this same group then published a retrospective study with 50 patients who received valganciclovir, reporting a median OS (mOS) of 25.0 months for all patients, 30.1 months in patients treated for at least 6 months, and 56.4 months in patients under continuous valganciclovir therapy (3). The basis for these clinical studies were data that indicated the protein and DNA for the cytomegalovirus (CMV) could be detected in almost all GBMs (4-6), and that survival was $>18$ months in GBMs with lower levels of CMV infection (5). These clinical results were very interesting to both neuro-oncology specialists and GBM patients. However, there has been significant debate since these publications, with certain groups suggesting CMV is not present in GBM $(7,8)$ and other groups contending it is $(4,9)$. Moreover, the analysis of the retrospective study has come into question, with concern raised over a type of selection bias referred to as 'immortal time bias' (10). Immortal time bias is a prejudice, as it refers to an amount of time in the follow-up period during which the outcome cannot occur due to the exposure definition. Thus, in the valganciclovir retrospective study, it would pertain to patients that had $\geq 6$ months of valganciclovir treatment, and within this group death cannot have occurred in the first 6 months of follow-up. However, Söderberg-Naucler et al repeated their analysis using Cox regression, which may prevent such bias, and still produced similar survival results in the valganciclovir group (11). 
With conflicting results regarding the presence of CMV within GBMs, the mechanism underlying the beneficial effect of valganciclovir on survival remains unknown; however, given the impressive improvement in survival of newly diagnosed GBM patients, the evaluation of valganciclovir in the recurrent setting is warranted.

We herein report a single-institution, retrospective analysis of GBM patients treated at recurrence with valganciclovir plus bevacizumab. Progression-free survival (PFS) and OS were compared to an institutional cohort for bevacizumab at recurrence and a small survival advantage was observed in patients treated in the recurrent setting with valganciclovir plus bevacizumab.

\section{Materials and methods}

Study design. Following approval by the Vanderbilt Institutional Review Board, we performed a retrospective chart review of all the patients treated for recurrent GBM at the Vanderbilt Neuro-Oncology clinic. We identified 13 patients who received treatment with both bevacizumab and off-label valganciclovir between August 1, 2013 and December 31, 2014, and 8 patients who were started on valganciclovir and bevacizumab concurrently. All patients but one (gliosarcoma) had a pathological diagnosis of GBM. These patients received bevacizumab at $10 \mathrm{mg} / \mathrm{kg}$ every 2 weeks and valganciclovir starting with a loading dose of $900 \mathrm{mg}$ b.i.d. for 21 days and then $450 \mathrm{mg}$ b.i.d. as maintenance therapy.

For the control cohort, we searched the database for all patients who were treated for first recurrence with GBM between January 1, 2005 and December 31, 2014, and identified 50 patients who met the following inclusion criteria: i) Had never received valganciclovir, even in later recurrences; ii) only first recurrence as to avoid any selection bias; iii) only a diagnosis of GBM; iv) were treated with concurrent radiation therapy and temozolomide followed by temozolomide therapy prior to recurrence; iv) recurrence occurred after 8 weeks from completion of chemoradiation; vi) had not had a second surgery for tumor progression; vii) had not been on a clinical trial at any point during their treatment; and viii) had signed consent forms to participate in a database containing all clinical information and tissue banking. A large portion of these control subjects received a chemotherapy drug in addition to bevacizumab.

Statistical analysis. Continuous variables were summarized using the median (range). Categorical variables were reported as frequencies and percentages of treatment group. PFS was defined as the time from therapy initiation to disease progression or death from any cause. OS was defined as the time from therapy initiation to death from any cause. All the patients had progressed as of the last follow-up. Patients who remained alive at the last follow-up were censored in the OS analysis. The distributions of PFS and OS were estimated using the Kaplan-Meier method and compared between treatment groups using the log-rank test.

Analysis of $\mathrm{O}^{6}$-methylguanine-DNA methyltransferase gene (MGMT) methylation and isocitrate dehydrogenase (IDH) mutation. Methylation of the MGMT promoter was detected using DNA isolated from formalin-fixed, paraffin-embedded specimens. Analysis was performed by methylation-specific polymerase chain reaction (PCR) using an ABI7900 PCR system (Applied Biosystems, Waltham, MA, USA). MGMT and $\beta$-actin copy numbers were used to calculate the ratio of $M G M T / \beta$-actin $\mathrm{x} 1,000$, and samples with scores $\geq 2.00$ were considered to be methylated. Analysis for mutations in $I D H 1$ and $I D H 2$ was performed using multiplex PCR coupled with a primer extension assay. Fluorescently labeled products were analyzed using capillary electrophoresis. The mutations tested included IDH1 R132H, R132 G, R132S, R132C, R132L, $\mathrm{R} 132 \mathrm{P}$ and the c.315C $>\mathrm{T}$ (G105G) polymorphism, as well as IDH2 R140G, R140W, R140Q, R140L, R140P, R172G, R172W, R172K, R172M, R172T, R172S and R172S.

\section{Results}

Patient characteristics. A total of 13 patients were identified, who had a first recurrence of high-grade GBM and were exposed to both bevacizumab and valganciclovir (Table I). Of these 13 patients, 8 were started concurrently on bevacizumab plus valganciclovir at recurrence, whereas the remaining 5 were started on bevacizumab at 3, 4, 6,9 and 12 months prior to valganciclovir initiation and prior to a second recurrence (1st recurrence as the start date of bevacizumab administration). The median exposure to valganciclovir was 9 months for all 13 patients (range, 3-16 months); the median exposure to valganciclovir was 9.5 months for the 8 concurrently treated patients (range, 3-16 months (Table I). Of the 13 patients, 12 had a pathological diagnosis of GBM, whereas 1 patient was diagnosed with gliosarcoma. All 13 patients were $I D H$ wild-type and $29 \%$ had $M G M T$ promoter methylation. The median Karnofsky performance status score was $80 \%$ and the median patient age was 61 years. Other chemotherapies used during treatment (at further disease progression) included 2 patients treated with lomustine and 6 treated with vorinostat.

Clinical results. There was a significant difference in PFS (log-rank $\mathrm{P}=0.054)$ and $\mathrm{OS}(\log$-rank $\mathrm{P}=0.005)$ in the 13 patients treated with valganciclovir plus bevacizumab compared with the bevacizumab control cohort, with a 6-month PFS (PF6) of 62\% [95\% confidence interval (CI): 0.40-0.95] and a mOS of 13.1 months [95\% CI: 9.13-not applicable (NA)] (Fig. 1A and B). For 8 of the 13 patients who were started concurrently on valganciclovir plus bevacizumab at first recurrence, the PF6 was 50\% (95\% CI: 0.25-1.0) and the mOS 11.3 months (95\% CI: 9.1-NA); neither of these reached significance compared with the control group in terms of PFS (log-rank $\mathrm{P}=0.509)$ and $\mathrm{OS}(\log$-rank $\mathrm{P}=0.107)$. For the institutional bevacizumab cohort, the PF6 was $34 \%$ (95\% CI: 0.23-0.50) and the mOS 8.7 months (95\% CI: 6.8-10.8) (Fig. 2A and B). Our institutional bevacizumab cohort mOS ( 8.7 months) was similar to historical data with bevacizumab, with a mOS ranging from 8.7 to 9.2 months $(12,13)$.

Of the 13 patients who were administered valganciclovir in our study, none developed leukopenia, skin reactions or nausea; however, 4 patients developed thrombocytopenia (platelet count $<100,000 / \mathrm{mcl}$ ), 3 of whom only had only one laboratory value $<100,000 / \mathrm{mcl}$, whereas 1 patient had $>1$ laboratory readings $<100,000 / \mathrm{mcl}$. 
Table I. Patient characteristics.

\begin{tabular}{lccc}
\hline Characteristics & $\begin{array}{c}\text { Valganciclovir, total } \\
\text { exposed with bevacizumab } \\
(\mathrm{n}=13)\end{array}$ & $\begin{array}{c}\text { Valganciclovir started } \\
\text { concurrently with bevacizumab } \\
(\mathrm{n}=8)\end{array}$ & $\begin{array}{c}\text { Bevacizumab } \\
\text { cohort } \\
(\mathrm{n}=50)\end{array}$ \\
\hline $\begin{array}{l}\text { Age, median (range), years } \\
\text { Gender (\%) }\end{array}$ & $61(33-77)$ & $58.5(49-77)$ & $58(18-75)$ \\
Male & 57 & 75 & 52 \\
Female & 43 & 25 & 48 \\
KPS score, median (range) & $80(60-90)$ & $80(60-90)$ & $80(50-100)$ \\
Received chemoradiation & 100 & 100 & 100 \\
with temozolomide (\%) & 29 & 37.5 & $\mathrm{a}$ \\
$M G M T$ methylated (\%) & 0 & 0 & $\mathrm{a}$ \\
IDH mutated (\%) & $9(3-16)$ & $9.5(3-16)$ & N/A \\
Median exposure to & & & \\
valganciclovir (range), months & & & \\
\hline
\end{tabular}

${ }^{a}$ In the majority of the patients the $M G M T$ and $I D H$ status were not known. KPS, Karnosfsky performance status; $M G M T, \mathrm{O}^{6}$-methylguanine-DNA methyltransferase gene; $I D H$, isocitrate dehydrogenase gene.
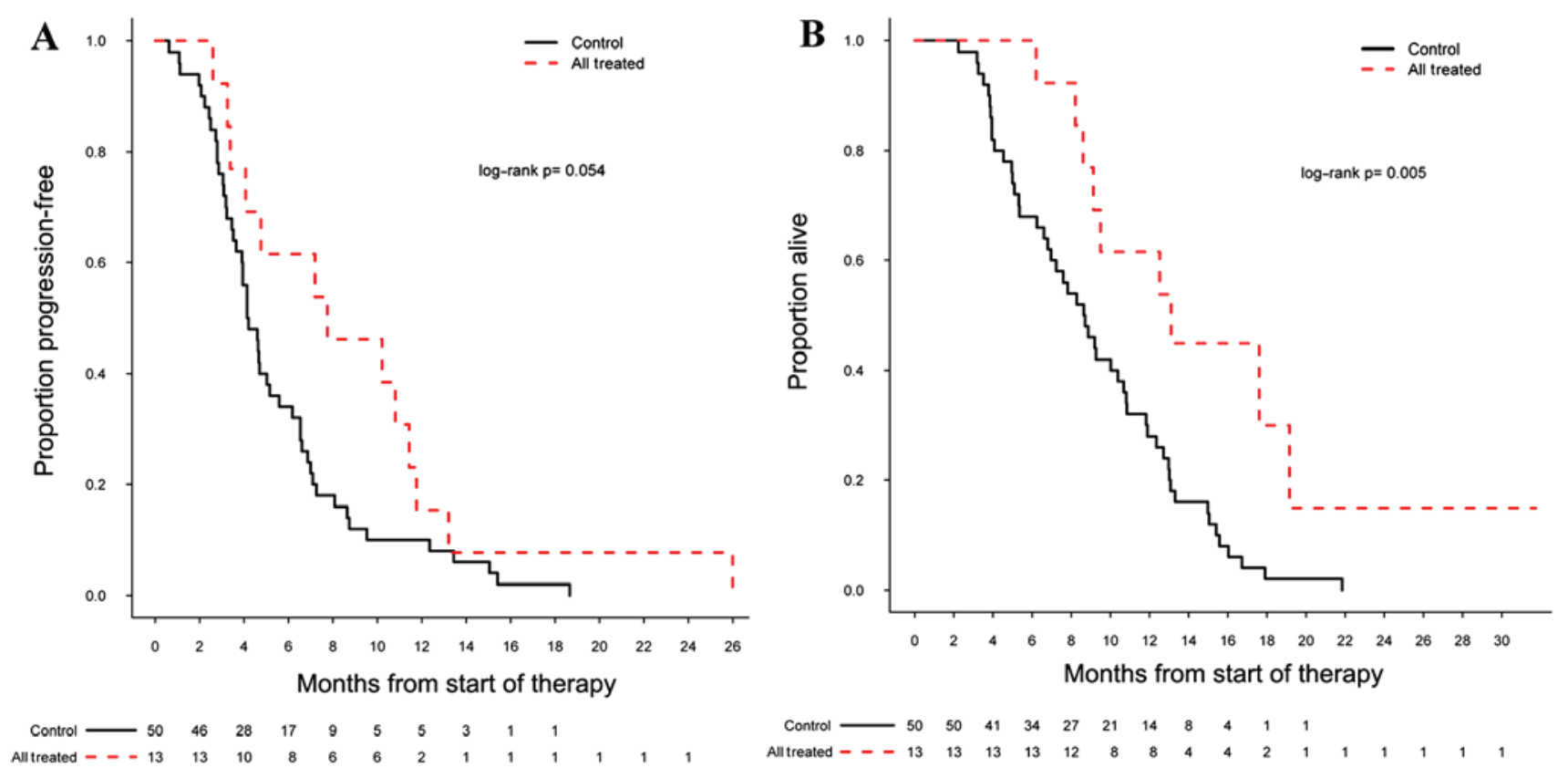

Figure 1. Kaplan-Meier survival curves for all patients. (A) Progression-free survival and (B) overall survival of all 13 patients treated with valganciclovir + bevacizumab vs. the control (bevacizumab alone) group.

\section{Discussion}

The treatment options for recurrent GBM are extremely limited and the field of neuro-oncology urgently requires additional therapies to supplement bevacizumab. The addition of valganciclovir to the standard-of-care in newly diagnosed GBM patients has shown promise in one retrospective study (3); however, this result and the presence of CMV in GBM remains highly controversial.

At our institution, 13 patients with recurrent GBM were placed on valganciclovir, of whom 8 were concurrently placed on bevacizumab therapy at first recurrence, whereas the remaining 5 had valganciclovir added to their existing bevacizumab regimen. In this study, we observed a small benefit in terms of OS in patients treated with valganciclovir plus bevacizumab compared with the bevacizumab cohort. In addition, the use of valganciclovir with bevacizumab appeared to be well tolerated in this setting.

We did not evaluate the presence of CMV protein in these patients, due to the controversy regarding its detection. Regardless, the question of whether valganciclovir prolongs survival when combined with bevacizumab is a major issue and, to the best of our knowledge, this study is the first to investigate valganiclovir in the recurrent setting. 

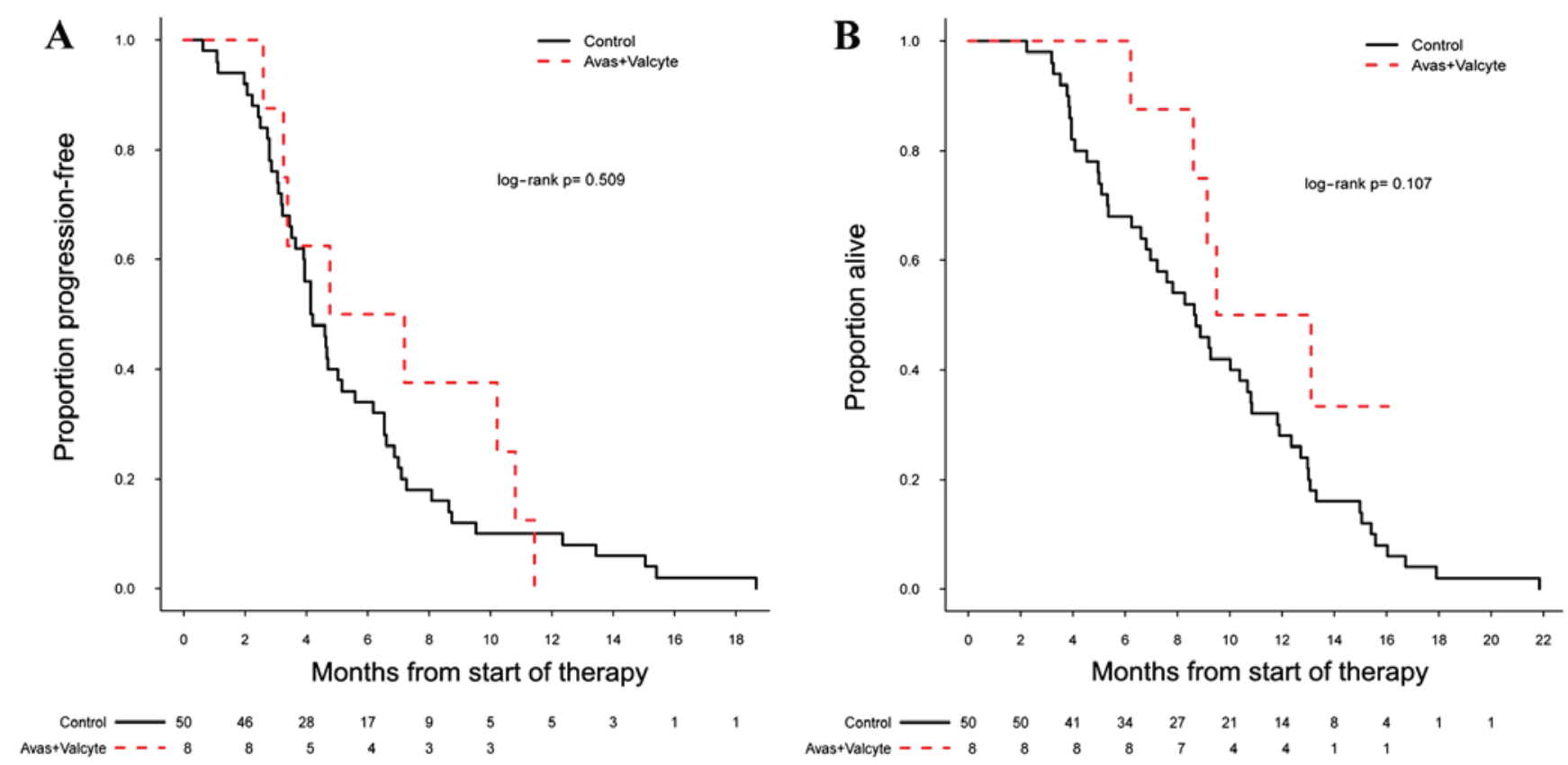

Figure 2. Kaplan-Meier survival curves for the concurrently treated group. (A) Progression-free survival and (B) overall survival for the 8 patients who were started on valganciclovir + bevacizumab at the same time vs. the control (bevacizumab alone) group.

If CMV is indeed present in these tumors, as several groups have reported, the mechanism of action of valganciclovir is likely associated with its inhibition of CMV replication and, thus, of CMV oncogene drivers (14). However, if CMV is not present in GBM, or is present in amounts not sufficient to affect tumor biology, the mechanism underlying the beneficial effect of valganciclovir on survival remains unclear, as other targets have not been identified. If indeed valganciclovir affects survival in these tumors, it appears to require an extended period of time ( $>6$ months) to observe an appreciable clinical difference (2); in the small group we present in this study, the median exposure was 9 months. Such a delayed effect is reminiscent of epigenetic modifiers, such as the DNA methyltransferase inhibitors used in the treatment of myelodysplastic syndromes, where $\geq 6$ months of treatment are required before observing a benefit (15). Thus, valganciclovir may inhibit GBM growth in a CMV-independent manner.

We observed that the addition of valganciclovir to bevacizumab led to a small increase in median OS in the recurrent setting. However, this study is limited by its retrospective nature and by the small number of patients that concurrently received valganciclovir plus bevacizumab. As a group, there was a significant increase in $\mathrm{mOS}$, but in the 8 concurrently treated patients the survival benefit did not reach significance. Moreover, the 5 patients who had valganciclovir added to the existing bevacizumab treatment, had not yet had a second recurrence; thus, some selection bias is possible. Therefore, any conclusions drawn by this study must be tempered and a larger prospective study is required to further evaluate these data. Additional clinical studies should be conducted in newly diagnosed patients where the time-to-treat is usually $>6$ months. Given the significant survival benefit reported in newly diagnosed GBM patients and the small trend we present here, coupled with the great need for improvement in this field, the risk-to-benefit ratio of such a study would be small.

\section{Acknowledgements}

Dr Clark is supported by a Vanderbilt Clinical Oncology Research Career Development Program K-12 Grant.

\section{References}

1. Stupp R, Mason WP, van den Bent MJ, Weller M, Fisher B, Taphoorn MJ, Belanger K, Brandes AA, Marosi C, Bogdahn U, et al: Radiotherapy plus concomitant and adjuvant temozolomide for glioblastoma. N Engl J Med 352: 987-996, 2005.

2. Stragliotto G, Rahbar A, Solberg NW, Lilja A, Taher C, Orrego A, Bjurman B, Tammik C, Skarman P, Peredo I and Söderberg-Nauclér C: Effects of valganciclovir as an add-on therapy in patients with cytomegalovirus-positive glioblastoma: A randomized, double-blind, hypothesis-generating study. Int J Cancer 133: 1204-1213, 2013.

3. Söderberg-Nauclér C, Rahbar A and Stragliotto G: Survival in patients with glioblastoma receiving valganciclovir. N Engl J Med 369: 985-986, 2013.

4. Cobbs CS, Harkins L, Samanta M, Gillespie GY, Bharara S, King PH, Nabors LB, Cobbs CG and Britt WJ: Human cytomegalovirus infection and expression in human malignant glioma. Cancer Res 62: 3347-3350, 2002.

5. Rahbar A, Stragliotto G, Orrego A, Peredo I, Taher C, Willems J and Söderberg-Naucler C: Low levels of human cytomegalovirus infection in glioblastoma multiforme associates with patient survival; -a case-control study. Herpesviridae 3: 3, 2012.

6. Bhattacharjee B, Renzette N and Kowalik TF: Genetic analysis of cytomegalovirus in malignant gliomas. J Virol 86: 6815-6824, 2012.

7. Baumgarten P, Michaelis M, Rothweiler F, Starzetz T, Rabenau HF, Berger A, Jennewein L, Braczynski AK, Franz K, Seifert $\mathrm{V}$, et al: Human cytomegalovirus infection in tumor cells of the nervous system is not detectable with standardized pathologico-virological diagnostics. Neuro Oncol 16: 1469-1477, 2014.

8. Hellstrand K, Martner A and Bergström T: Valganciclovir in patients with glioblastoma. N Engl J Med 369: 2066, 2013.

9. Ranganathan P, Clark PA, Kuo JS, Salamat MS and Kalejta RF: Significant association of multiple human cytomegalovirus genomic loci with glioblastoma multiforme samples. J Virol 86: 854-864, 2012. 
10. Liu CJ and $\mathrm{Hu} \mathrm{YW}$ : Immortal time bias in retrospective analysis: Is there a survival benefit in patients with glioblastoma who received prolonged treatment of adjuvant valganciclovir? Int $\mathbf{J}$ Cancer 135: 250-251, 2014.

11. Söderberg-Naucler C, Peredo I, Rahbar A,Hansson F, Nordlund A and Stragliotto G: Use of Cox regression with treatment status as a time-dependent covariate to re-analyze survival benefit excludes immortal time bias effect in patients with glioblastoma who received prolonged adjuvant treatment with valganciclovir. Int J Cancer 135: 248-249, 2014.

12. Nghiemphu PL, Liu W, Lee Y, Than T, Graham C, Lai A, Green RM, Pope WB, Liau LM, Mischel PS, et al: Bevacizumab and chemotherapy for recurrent glioblastoma: A single-institution experience. Neurology 72: 1217-1222, 2009.
13. Friedman HS, Prados MD, Wen PY, Mikkelsen T, Schiff D, Abrey LE, Yung WK, Paleologos N, Nicholas MK, Jensen R, et al: Bevacizumab alone and in combination with irinotecan in recurrent glioblastoma. J Clin Oncol 27: 4733-4740, 2009.

14. Cobbs CS: Cytomegalovirus and brain tumor: Epidemiology, biology and therapeutic aspects. Curr Opin Oncol 25: 682-688, 2013.

15. Garcia-Manero G and Fenaux P: Hypomethylating agents and other novel strategies in myelodysplastic syndromes. J Clin Oncol 29: 516-523, 2011. 